Qushmua E. Alzahrani, Gary G. Adams, Richard B. Gillis, Tabot M.D. Besong, M. Samil Kök, Emily Fong, Richard A. Harding, Jan E.G. van Dam, Richard J.A. Gosselink*, Arthur J. Rowe and Stephen E. Harding*

\title{
Matrix-free hydrodynamic study on the size distribution and conformation of three technical lignins from wood and non-wood
}

\begin{abstract}
Molecular weight (MW) and related conformational data of three commercially available technical lignins (Alcell L, kraft L, and soda L) have been studied by means of analytical ultracentrifugation, taking advantage of some recent developments in both sedimentation velocity and sedimentation equilibrium determinations. The lignins were dissolved in dimethyl sulphoxide (with ca. $90 \%$ solubility), and solutions were studied with regards to their oligomeric state, heterogeneity profiles (distribution of sedimentation coefficients), and molecular weight distributions (MWD). Alcell L and soda L have similar properties showing one major low MW component and two minor high MW components, whereas kraft L appears to be larger and more uniform, i.e., it shows a more monodisperse MWD. Weight average molecular weight $\left(M_{\mathrm{w}}\right)$ data from sedimentation equilibrium obtained by the new SEDFIT-MSTAR procedure in conjunction with MULTISIG analysis were found to be $\sim 18 \mathrm{kDa}$ (Alcell L), $25 \mathrm{kDa}$ (kraft L), and $15 \mathrm{kDa}$ (soda L). Further analysis of the data
\end{abstract}

*Corresponding authors: Richard J.A. Gosselink Food and Biobased Research, Wageningen UR, Bornse Weilanden 9, NL-6708 WG Wageningen, The Netherlands e-mail: richard.gosselink@wur.nl. http://orcid.org/0000-0001-7733-6109; and Stephen E. Harding, National Centre for Macromolecular Hydrodynamics, School of Biosciences, University of Nottingham, College Road, Sutton, Bonington LE12 5RD, UK, e-mail: steve.harding@nottingham.ac.uk Qushmua E. Alzahrani and Gary G. Adams: National Centre for Macromolecular Hydrodynamics, School of Biosciences, University of Nottingham, College Road, Sutton, Bonington LE12 5RD, UK; and Faculty of Medicine and Health Science, University of Nottingham, Queens Medical Centre, Nottingham NG7 2RD, UK

Richard B. Gillis, Tabot M.D. Besong, Emily Fong, Richard A. Harding and Arthur J. Rowe: National Centre for Macromolecular Hydrodynamics, School of Biosciences, University of Nottingham, College Road, Sutton, Bonington LE12 5RD, UK

M. Samil Kök: National Centre for Macromolecular Hydrodynamics, School of Biosciences, University of Nottingham, College Road, Sutton, Bonington LE12 5RD, UK; and Department of Food Engineering, Abant Izzet Baysal University, 14280 Bolu, Turkey Jan E.G. van Dam: Food and Biobased Research, Wageningen UR, Bornse Weilanden 9, NL-6708 WG Wageningen, The Netherlands by means of the routines MULTISIG and M_INVEQ confirmed the presence of additional components in Alcell $\mathrm{L}$ and soda $\mathrm{L}$, and the larger size and high degree of monodispersity of kraft L. The intrinsic viscosity data of the three lignins were found to be very similar in the range of 22-24 $\mathrm{ml} \mathrm{g}^{-1}$, and all data were consistent with an elongated plate shape molecular structure with an equivalent discoid aspect ratio $\sim 30$.

Keywords: Alcell L, analytical ultracentrifugation, disc shape, DMSO, kraft L, soda L, viscometry

DOI 10.1515/hf-2014-0318

Received October 24, 2014; accepted March 27, 2015; previously published online $x x$

\section{Introduction}

Lignins are a class of natural, highly branched phenylpropanoid macromolecules that have a random and amorphous three-dimensional structure, in which the partly cross-linked chains are hydrophobic, heterogeneous, and polydisperse. The carbon content of the aromatic lignins is around $60-63 \%$, i.e., higher than that of the accompanying polysaccharides (Sarkanen and Ludwig 1971; Fengel and Wegener 1984). Lignins are classified according to the composition of the three major phenylpropan units, namely 4-hydroxy-, guaiacyl-, and syringyl-type phenylpropanes, abbreviated as $\mathrm{H}, \mathrm{G}$, and $\mathrm{S}$ units, respectively. As is well known, there are in essence G-lignins (in softwoods), GS-lignins (in hardwoods), and HGS lignins (in grasses) (Sarkanen and Ludwig 1971; Fengel and Wegener 1981; Zhong and Ye 2007; Vanholme et al. 2010).

Lignins are available either as an alkaline aqueous solution or in isolated powder form (Käuper 2004). Alcell L (and organosolv type L), kraft L (also called sulphate L), and soda L (also called alkali L) are three examples of 
technical lignins, which are obtained by different processes (Garver and Callaghan 1991; De Martino 2005). Kraft lignin is extracted from wood in the presence of alkaline sodium sulphide, which is the most common pulping process nowadays. Alcell lignin is separated from wood by acidic ethanol-water digestion, and it can be considered as an organosolv-type pulping process not yet applied at an industrial level (Kubo and Kadla 2004; Lallave et al. 2007). Soda lignin is a product of the alkaline pulping of annual plants (straw, for example) (Käuper 2004).

The molecular weight (MW) of industrial lignins is an important parameter for their utilisation as an aromatic material for various purposes (Dong and Fricke 1995; Brebu and Vasile 2010). The MW and MW distribution (MWD) vary strongly depending on the tree/plant species and the type of chemical processing for their isolation. MWs are expressed usually on a weight average basis $\left(M_{\mathrm{w}}\right)$, but also as a number average $\left(M_{\mathrm{n}}\right)$ or as a z-average $\left(M_{\mathrm{z}}\right)$. These data are, however, not easily measured because of the polydispersity and incomplete solubility of lignins, which are generally insoluble in aqueous solvent. Only lignosulphonates are water soluble. Common lignin solvents are alkali, dimethyl sulphoxide (DMSO), dioxane/ water (9:1), acetone/water, and dimethylformamide.

As a routine, MW and MWD are determined by size exclusion chromatography (SEC), where the MW data are related to calibration standards of known MW. In an ideal case, the standards should have a very similar conformation to that of the target polymer; however, this prerequisite is not easy to realize. Sulphonated polystyrene standards are in special cases well suited to this purpose (Baumberger et al. 2007; Gosselink et al. 2010). Nevertheless, the SEC approach has many pitfalls.

Analytical ultracentrifugation has been successfully tested in the past on lignins (Goring 1962; Sarkanen et al. 1981, 1982, 1984). It is a technique that combines both an inherent separation and analysis ability without the need for a separation matrix (i.e., columns or membranes). Nevertheless, this method has been seldom applied, mainly due to inaccessibility of instrumentation and complexity of analysis. There has been, however, significant recent progress in the field of analytical ultracentrifugation that renders it possible to obtain profiles and accurate absolute MW data on a fairly routine basis (Harding 2005; Harding et al. 2011), as commercial instrumentation is now widely available. Furthermore, the development of the new SEDFIT-MSTAR (Schuck et al. 2014) and MULTISIG (Gillis et al. 2013) software facilitates the data evaluation.

The aim of the present study is the determination of MW-related data of three technical lignins from different sources, which have been solubilised in DMSO. The focus is on the absolute MWs, MWDs, oligomeric state, and heterogeneity profiles, and the conformation of the lignins in solution. The utility and complementarity of the two types of analytical ultracentrifuge experiment (sedimentation velocity and sedimentation equilibrium) for heterogeneity and MW analysis will be demonstrated. Garver and Callaghan (1991) studied the conformation of lignins on the basis of measurement of translational diffusion coefficients and found oblate (disc or plate shape) structures of high aspect ratio. The intention is to take advantage of the availability of the latest software for the analysis of hydrodynamic conformation (Harding et al. 2005; García de la Torre and Harding 2013) to explore the issue of lignin conformation further.

\section{Materials and methods}

Alcell L (a GS lignin) is an organosolv-type lignin produced from mixed hardwoods by Repap Enterprises Inc. (Montreal, Canada). The softwood kraft L (a G lignin) is the product Indulin AT produced by MeadWestvaco (Richmond, VA, USA). Soda L (P1000, a HGS lignin) is produced from a mixture of wheat straw and sarkanda grass by GreenValue SA (Orbe, Switzerland).

Each lignin sample was dissolved in a solvent consisting of $90 \%$ (w/v) DMSO and 10\% (w/v) phosphate-chloride buffer ( $\mathrm{pH}$ 7.0) with an ionic strength of $0.1 \mathrm{~mol} \mathrm{l}^{-1}$. Dispersions were mixed on a roller mixer at room temperature overnight. Undissolved material (ca. 10\%) was removed by centrifugation at 6,000 rpm (approximately $5000 \mathrm{~g}$ ). Final concentrations after clarification were assessed by an Atago Co. (Tokyo, Japan) DD-7 differential refractometer and a refractive index increment of $0.218 \mathrm{ml} \mathrm{g}^{-1}$ (Gupta and Goring 1960).

Sedimentation velocity in the analytical ultracentrifuge was performed in a Beckman (Palo Alto, CA, USA) XL-I ultracentrifuge. Lignin solutions $(\sim 400 \mu \mathrm{l})$ at a concentration of $\sim 0.2 \mathrm{mg} \mathrm{ml}^{-1}$ and $90 \%$ DMSO were injected into the sample and reference channels, respectively, of $12 \mathrm{~mm}$ optical path length cells. The balanced cells were then loaded into an analytical eight-hole titanium rotor An50-Ti and placed in the rotor chamber of the analytical ultracentrifuge. The Rayleigh interference optical system was used for recording concentration profiles and the movement of the sedimentation boundary in the analytical ultracentrifuge cell (Harding 2005). For checking for the presence of aggregates, an initial low rotor speed of $3000 \mathrm{rpm}$ was employed, before adjusting to a final rotor speed of 45,000 rpm. Scans were taken at $2 \mathrm{~min}$ intervals for a run time of $\sim 24 \mathrm{~h}$. The data were analysed by means of the SEDFIT algorithm of Dam and Schuck (2004), which gives a distribution of sedimentation coefficient, $s$, and provides an assessment of the polydispersity. The " $c(s)$ vs. $s$ " distribution method of SEDFIT was applied, rather than the ls-g(s) distribution, because of the very low $s$ and incomplete resolution of the sedimenting boundaries from the air-solution meniscus, where $s$ is in seconds or Svedberg units $\mathrm{S}$, where $1 \mathrm{~S}=10^{-13} \mathrm{~s}$. The standard conditions of density and viscosity of water at $20.0^{\circ} \mathrm{C}$ served as a basis for adjustment of the (weight average) sedimentation coefficients of resolved peaks $s$ to $s_{20, w}$ values (Schachman 1992). Because of the very low concentrations employed $\left(\sim 0.2 \mathrm{mg} \mathrm{ml}^{-1}\right)$, correction 
for non-ideality (extrapolation to infinite dilution) was not necessary, and the assumption $S^{0}{ }_{20, \mathrm{w}} \sim S_{20, \mathrm{w}}$ is reasonable. A value for the partial specific volume $\bar{v}$ of $0.61 \mathrm{ml} \mathrm{g}^{-1}$ was used (Rubio et al. 1979). The $s^{0}{ }_{20, \mathrm{w}}$ value obtained will be a function of the size (MW), shape, and degree of solvent association or "hydration" of the macromolecule.

Sedimentation equilibrium was also performed in the Beckman XL-I ultracentrifuge (Svedberg and Pedersen 1940; Creeth and Pain 1967), but at a lower rotor speed of 20,000 rpm. At lower rotor speeds, the centrifugal force is balanced by diffusive forces in the opposite direction until a final steady state is achieved, which is a function only of MW and related parameters, i.e., it gives an absolute measure of MW and MWD. Cell filling and solvents were as for sedimentation velocity, but the injection volumes $(\sim 80 \mu \mathrm{l})$ were lower to reduce the time to equilibrium. Rayleigh interference optics were used. Initial scans (an average of five) were taken as soon as 20,000 rpm was attained, and these were then subtracted from the final equilibrium scans (an average of five) to correct for anomalies through any window distortion, etc. (Ang and Rowe 2010). The final "equilibrium concentration vs. radial displacement" profiles were then analysed by SEDFIT-MSTAR (Schuck et al. 2014), yielding (i) an accurate estimate for the apparent $M_{\mathrm{w}}$, i.e., $M_{\mathrm{w}, \mathrm{app}}$, for the whole distribution based on both the $M^{\star}$ function of Creeth and Harding (1982) and the "hinge point method" (Schuck et al. 2014); (ii) point or local apparent weight average MWs, i.e., $M_{\text {wapp }}(r)$, as a function of radial position $r(\mathrm{~cm})$ and hence concentration $c(r)$ [from the $c(r)$ vs. $r$ profiles] in the ultracentrifuge cell. As already pointed out, the loading concentrations $\left(0.2 \mathrm{mg} \mathrm{ml}^{-1}\right)$ were low to minimize the effects of non-ideality. Under these conditions, the $M_{\text {w.app }}$ is approximately the "ideal" $M_{\mathrm{w}}$, i.e., $M_{\text {w,app }} \sim M_{\mathrm{w}}$, and $M_{\text {w,app }}(r) \sim M_{\mathrm{w}}(r)$.

Further analyses of the experimental equilibrium distributions were then carried out based on MULTISIG and M_INVEQ software. The former provides a means for the definition of MWD in polydisperse systems by sedimentation equilibrium analysis (Gillis et al. 2013), complementing the $M_{\mathrm{w}}$ for the whole distribution obtained from SEDFIT-MSTAR. MULTISIG also makes possible the computation of point-averaged MWs at a series of radial positions, via a small extension (known as MULTISIG_RADIUS). This approach has found recent application by Nikolajski et al. (2014), dealing with the reversible self-association of an aminocellulose. These methods have been employed as published except that from an initial MULTISIG fit, an average baseline offset $(E)$ was computed, and this value was fixed for the subsequent application of both SEDFIT-MSTAR and MULTISIG_RADIUS. This procedure produced "smoother" data sets of $M_{\text {w.app }}(r)$ vs. radial position $r$ [or $c(r)$ ] compared with that from SEDFIT-MSTAR. The system also provides point number-averaged MW, i.e., $M_{\mathrm{n}}(r)$, and point z-averaged MW, i.e., $M_{\mathrm{z}}(r)$.

MULTISIG software returns "reduced" molecular weight $(\sigma)$ data, where

$$
\sigma=M(1-\bar{v} \cdot \rho) \omega^{2} r / R T
$$

In Eq. (1), $\bar{v}$ is the partial specific volume of the solute, $\rho$ the density of the solvent, $\omega$ the angular velocity of the rotor, $r$ the radial position, $R$ the gas constant, and $T$ is the temperature of the rotor (K). The $\sigma$ values are converted into conventional molecular weight values via the software SEDNTERP (Laue et al. 1992). This data set was then analysed by the programme SIGSTAR, equivalent to the established routine MSTAR within SEDFIT-MSTAR (Schuck et al. 2014), but specifically applied to the processed data within MULTISIG as a consistency check via the weighted average $\sigma_{\mathrm{w}}$, which is averaged over the whole cell.

Finally, the sedimentation equilibrium data were analysed by the programme M_INVEQ to explore further the nature of the distribution of molecular weights in the Alcell $\mathrm{L}$ and soda $\mathrm{L}$ samples. Both lignins have a rather wide distribution, which appears to be a possible sum of two components, with a $\approx 1: 2$ ratio. This programme is in essence a minor modification of an existing routine (INVEQ reviewed in Rowe 2011). M_INVEQ yields estimates for the relative amounts of these two defined species in an equilibrium distribution.

Capillary viscometry, for relative viscosity measurements, $\eta_{\text {rel}}$, was performed in a Schott-Geräte Ostwald viscometer (for the theory, see Harding 1997 ) at $20.00 \pm 0.01^{\circ} \mathrm{C}$. Solution concentrations were in the range of $0.9-1.3 \mathrm{mg} \mathrm{ml}^{-1}$; because of the low concentrations, a density correction to the flow time measurements was not necessary (Harding 1997). Intrinsic viscosities $[\eta]$ were estimated at each concentration, $c\left(\mathrm{~g} \mathrm{ml}^{-1}\right)$, by the Solomon and Ciuta (1963) equation:

$$
[\eta] \simeq(1 / c) \cdot\left[2\left\{\eta_{\mathrm{sp}}-\ln \left(\eta_{\mathrm{rel}}\right)\right\}\right]^{1 / 2},
$$

and a mean value taken as the consensus $[\eta]$.

\section{Results and discussion}

\section{Heterogeneity and sedimentation coefficients (s)}

Sedimentation velocity in the analytical ultracentrifuge (Figure 1) shows that all three lignins have a distribution of $s$, which are broad for Alcell $L$ and soda $L$ but narrow for kraft $\mathrm{L}$. The former two have a main peak with a very low $s$ (within $0.35-0.55 \mathrm{~S}$ ) and two clear minor peaks at higher

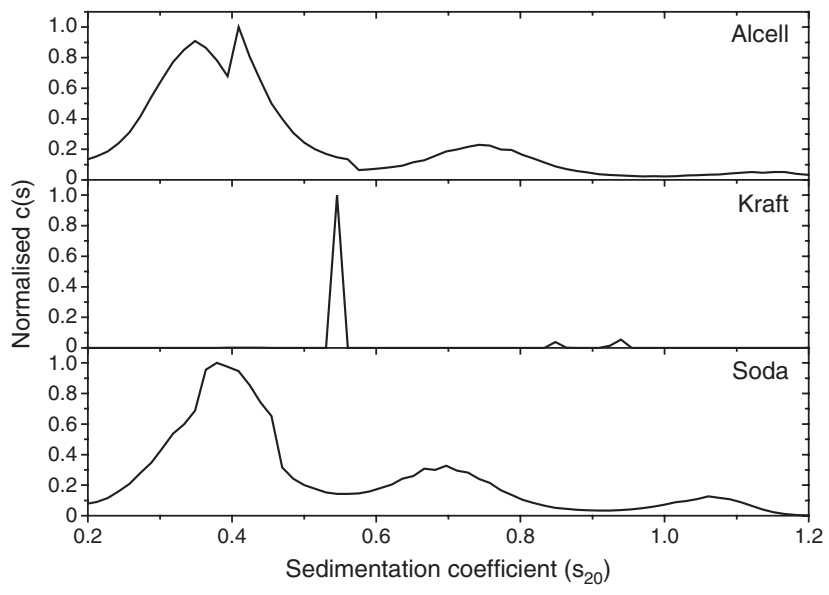

Figure 1: Sedimentation velocity of wood lignins.

Sedimentation coefficient distribution plots, $c(s)$ vs. $s$ from SEDFIT for Alcell lignin (top), kraft lignin (middle), and soda lignin (bottom) in $90 \%$ DMSO. At the concentrations used $\left(\sim 0.2 \mathrm{mg} \mathrm{ml}^{-1}\right)$, non-ideality effects can be assumed to be negligible. 
Table 1: Hydrodynamic data for Alcell, kraft, and soda lignins in 90\% DMSO.

\begin{tabular}{lrrrrrrr}
\hline Lignin & ${ }^{\mathrm{a}} \boldsymbol{M}_{\mathrm{w}}(\mathrm{kDa})$ & ${ }^{\mathrm{b}} \boldsymbol{M}_{\mathrm{w}}(\mathrm{kDa})$ & ${ }^{\mathrm{C}} \boldsymbol{M}_{\mathrm{w}}(\mathbf{k D a})$ & ${ }^{\mathrm{d}} \boldsymbol{S}_{20, \mathrm{w}}(\mathbf{S})$ & ${ }^{\mathrm{e}} \boldsymbol{S}_{20, \mathrm{w}}(\mathbf{S})$ & ${ }^{\mathrm{f}} \boldsymbol{S}_{20, \mathrm{w}}(\mathbf{S})$ & {$[\eta]\left(\mathrm{ml} \mathbf{g}^{-1}\right)$} \\
\hline Alcell & $19.3 \pm 1.0$ & 16.5 & 50.7 & 0.95 & 1.95 & 2.30 & $23.6 \pm 2.0$ \\
Kraft & $24.9 \pm 1.0$ & 27.2 & - & 1.25 & - & - & $22.7 \pm 0.8$ \\
Soda & $15.4 \pm 0.7$ & 10.3 & 30.2 & 0.91 & 1.66 & 2.20 & $22.0 \pm 0.3$ \\
\hline
\end{tabular}

aFrom sedimentation equilibrium, $M^{*}$ extrapolation method in SEDFIT-MStar; ${ }^{b}$ main component (from MultiSig/M_INVEQ); ${ }^{\mathrm{c} m i n o r}$ component (from MultiSig/M_INVEQ); ${ }^{d}$ main component; ${ }^{e}$ second component; fthird component.

$s$ (Table 1). The kraft L, in contrast, displays only a single significant peak.

\section{Weight average molecular weights $\left(M_{\mathrm{w}}\right)$}

The $M_{\mathrm{w}}$ for the whole distributions of the three lignins, as obtained by the SEDFIT-MSTAR procedure (Schuck et al. 2014), and the individual values obtained are given in Table 1, and the corresponding plots are presented in Figure 2.

SEDFIT-MSTAR reveals a near-linear plot of $\ln J(r)$ vs. $r^{2}$ for kraft L [where $J(r)$ is the local concentration (expressed in fringe units) at radial positions $r$ ]; however, evidence of upward curvature is seen for Alcell $\mathrm{L}$ and soda L, a feature of greater polydispersity. The " $M^{\star}$ vs. radius" plots (Figure 2 ) are contiguous and give accurate estimates for $M_{\mathrm{w}}$ (representing the whole MWD) from the identity $M_{\mathrm{w}}=M^{\star}(r=b)$ (Creeth and Harding 1982), where $b$ is the radial position at the cell base. The "point average $M_{\mathrm{w}}(r)$ vs. concentration" plots from SEDFIT-MSTAR are also presented in Figure 2. Despite the noise (deriving from the low fringe increments due to the low concentrations), plots of point average " $M_{\mathrm{w}}(r)$ vs. concentration $J(r)$ " (lower set of plots in Figure 2) as obtained from local slopes along the " $\ln J(r)$ vs. $r$ " curves show a characteristic increase in $M_{\mathrm{w}}(r)$ with $J(r)$ in the case of Alcell L and soda L. This is in agreement with data of sedimentation velocity, which show the presence of more than one significant component for these lignins (Figure 1). In the case of kraft
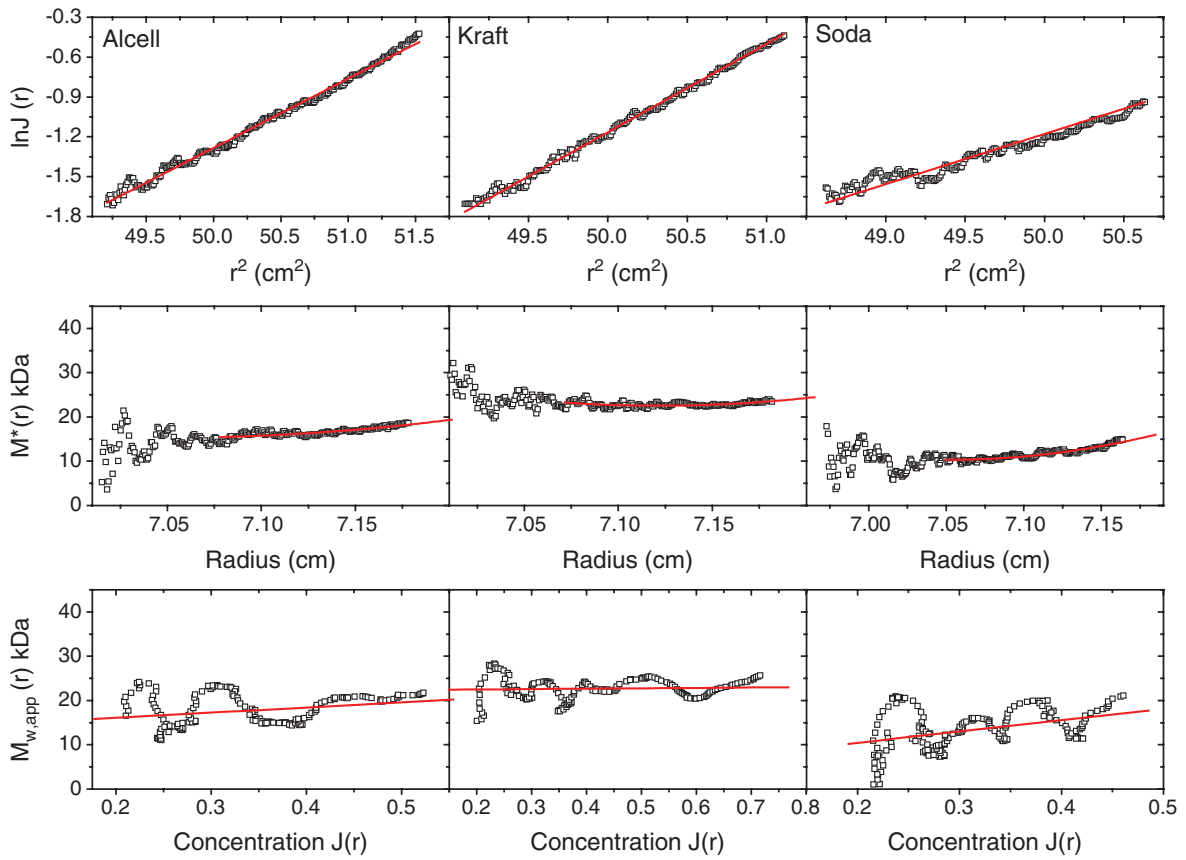

Figure 2: SEDFIT-MSTAR sedimentation equilibrium plots for Alcell (left), kraft (middle), and soda (right) lignins in $90 \%$ DMSO at a loading concentration of $\sim 0.2 \mathrm{mg} \mathrm{ml}^{-1}$.

Top row: log concentration, in fringe displacement units, $J(r)$, vs. the square of the radial displacement from the axis of rotation plot $r^{2}$, where $r(\mathrm{~cm})$ is the radial distance from the centre of rotation (open squares). Middle row: $M^{*}$ vs. $r$ plot (open squares) and fit. The value of $M^{*}$ extrapolated to the cell base $=M_{\text {w.app }}$, the apparent weight average molecular weight for the whole distribution. Bottom row: point or local apparent weight average molecular weight $M_{\mathrm{w}, \mathrm{app}}(r)$ at radial position $r$ (open squares) plotted against the local concentration in the ultracentrifuge cell. 
$\mathrm{L}$, no clear increase is observable, which commensurates with the unimodal distribution seen from sedimentation velocity (Figures 1 and 2).

To explore this behaviour further, more refined analyses of the sedimentation equilibrium data can be performed by means of MULTISIG and M_INVEQ, which provide quantitative information about the presence of different components, rather than averaging the data with SEDFIT-MSTAR. In Figure 3, the results are plotted on common axes. First, the data confirm (with a smoother fit) the point average data for $M_{\mathrm{w}}(r)$ from the SEDFIT-MSTAR routine and also yield estimates for $M_{\mathrm{n}}(r)$ and $M_{\mathrm{z}}(r)$. The plots are also in agreement with the observation that the average MW values are increasing steadily with increasing radii over the solution column for Alcell $\mathrm{L}$ and soda $\mathrm{L}$. This indicates for these two lignins a significant polydispersity.
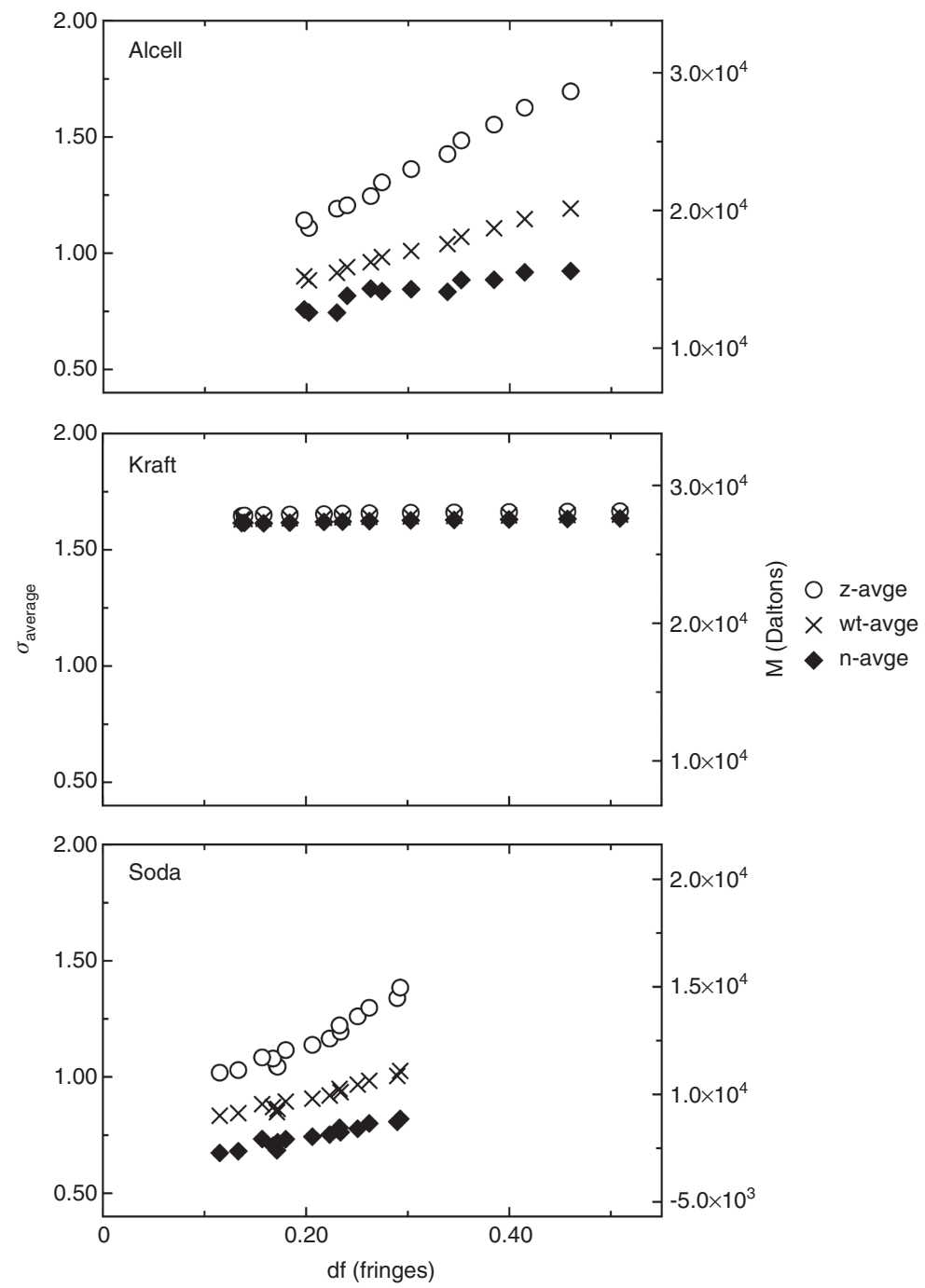

Figure 3: Plots of the number, weight, and $z$-average values from MULTISIG as a function of concentration (in fringe values) for the three samples. No line has been fitted through these values for lack of a hypothesis to support any particular model.
By contrast, the average values of kraft $\mathrm{L}$ are nearly constant with respect to radius, which is indicative of a relatively narrow MWD. M-INVEQ software resolves the MULTISIG distributions in terms of multi-modality. It yields two components (Figure 4) for Alcell L and soda $\mathrm{L}$, as presented in Table 1, and are consistent with the "unresolved" values from SEDFIT-MSTAR. It should be emphasised that the finding of two components in a mass ratio of approximately 1:2 does not imply necessarily any sort of monomer-dimer equilibrium. Quite the contrary, the general agreement between the $c(s)$ distributions of $s$ values and the distributions seen by MULTISIG and M_INVEQ analyses are arguments against such a hypothesis, and the components are not in a reversible equilibrium. This contrasts strongly with a recent demonstration of self-association behaviour in a class of cellulose 

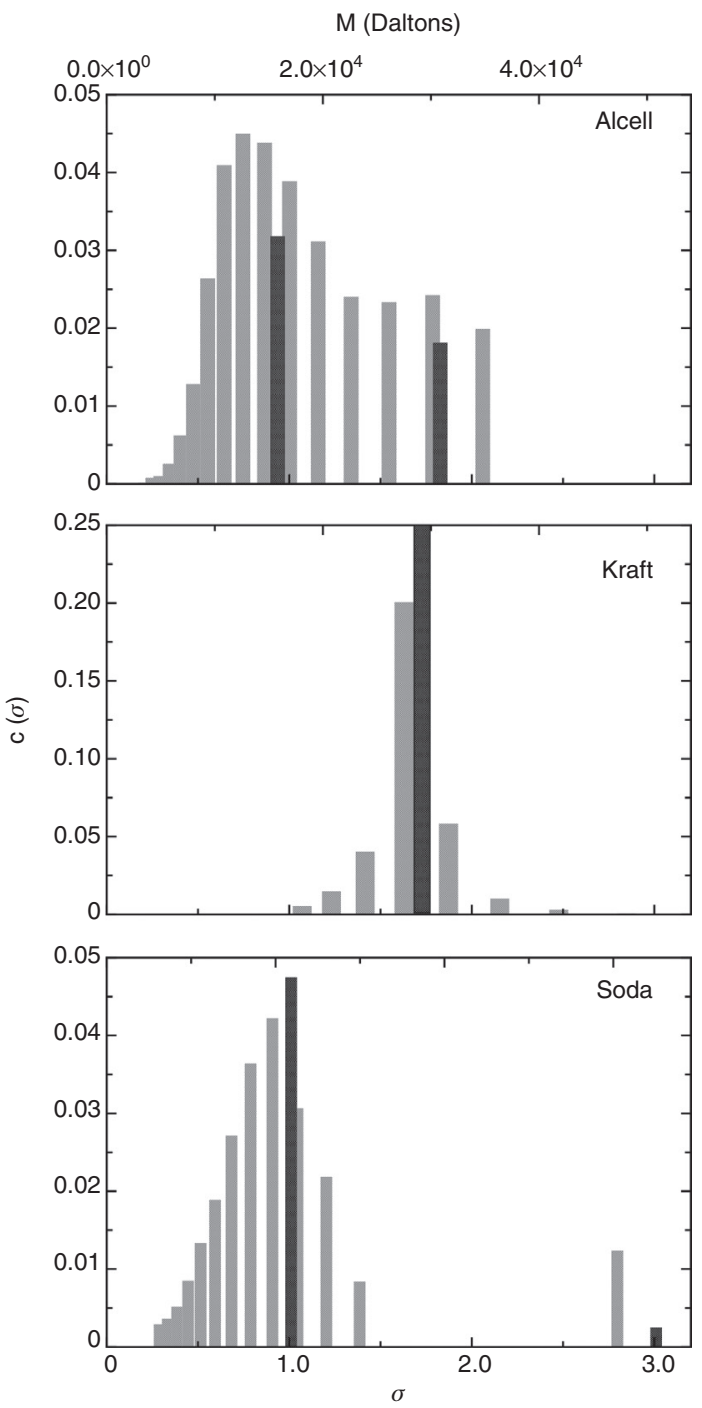

Figure 4: The distribution of molecular weight values as yielded by MULTISIG analysis (grey) and two-component M_INVEQ fit (black).

derivatives known as the aminocelluloses (Heinze et al. 2011; Nikolajski et al. 2014). This behaviour cannot be seen in kraft $\mathrm{L}$, where there is no resolution into two components, and this finding is once more consistent with the data of sedimentation velocity (Figure 1).

There are hybrid methods of MW determination based on a combination of (preparative) column chromatography, where the columns are "self-calibrated" by isolating fractions and measuring the MWs of the isolated fractions absolutely by sedimentation equilibrium (or light scattering). This approach was tested for carbohydrate polymers such as alginate (Ball et al. 1988) and dextran (Ball et al. 1990) and also for lignins (Sarkanen et al. 1982). Such procedures are less dependent from conformational differences between standards and specimens. Nevertheless, this method is comparatively laborious and requires multiple sedimentation equilibrium determinations and still requires the assumption of column inertness of chromatography columns.

Earlier reports have pointed out self-association behaviour of organsolv lignins in aqueous solvents (Sarkanen et al. 1982). The present study on Alcell L, kraft L, and soda $L$ effectively rules out the presence of a reversible interaction behaviour in 90\% DMSO. The increasing $M_{\mathrm{w}}(r)$ as a function of $c(r)$ seen by MULTISIG_RADIUS analysis appears to be due to polydispersity (non-interacting components of different molecular weight) rather than due to a self-association.

\section{Intrinsic viscosity}

Intrinsic viscosity data are presented in Figure 5a-c, which are all in the range of $22-24 \mathrm{ml} \mathrm{g}^{-1}$. These values are higher than those obtained by Dong and Fricke (1995) of $\sim 8 \mathrm{ml} \mathrm{g}^{-1}$ (obtained in dimethyl formaldehyde at $45.0^{\circ} \mathrm{C}$ ), probably because of the different solvent used. It is possible to estimate the extension or aspect ratio of the lignin molecules from the relation (Harding 1997)

$$
[\eta]=v \cdot v_{s},
$$

where $v_{\mathrm{s}}\left(\mathrm{ml} \mathrm{g}^{-1}\right)$ is the swollen specific volume (which takes into account solvation) and $v$ is the Einstein-Simha shape parameter (Simha 1940; Harding et al. 1982; Harding 1997). In Table 2, a value of $v$ is presented for three plausible values of $v_{\mathrm{s}}\left(0.6,1.0\right.$, and $\left.1.4 \mathrm{ml} \mathrm{g}^{-1}\right)$. The asymmetry of the three lignins solubilised in $90 \%$ DMSO can be estimated by translating $v$ into ellipsoidal aspect ratios. This can easily be done by the routine ELLIPS1 (Harding et al. 2005; García de la Torre and Harding 2013). The aspect ratio also depends on the type of ellipsoid of revolution, which can be prolate/rod shape or oblate/disc shape. So in Figure 5 both possibilities are presented in Figure $5 d$ the former and in Figure $5 \mathrm{e}$ the latter. The three lignins have approximately the same value for the Einstein-Simha shape factor; thus, the prolate and oblate shapes can be collectively used as models for all three lignins analysed.

Favis and Goring (1984) provided evidence that lignins may have oblate, flat disk structures, and this was later reinforced by measurements on acetylated lignins in deuterated chloroform by Garver and Callaghan (1991). If this is adopted as a working hypothesis for the measurements here in 90\% DMSO solutions, then plate-shape models would have an aspect ratio of $\sim 30: 1$ (Figure $5 d$ ). 

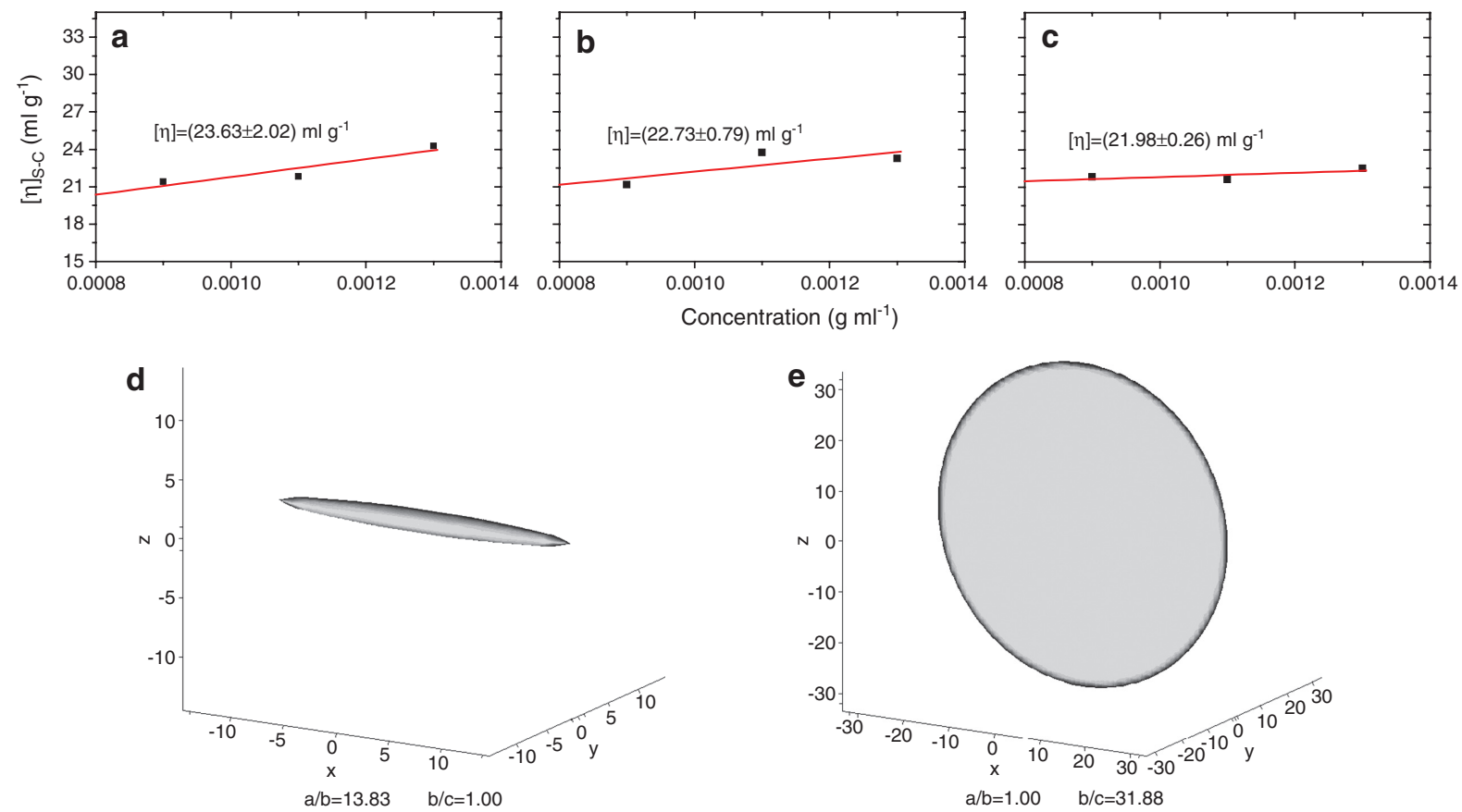

Figure 5: Intrinsic viscosity [from Eq. (2)] estimates at different concentrations for (a) Alcell lignin, (b) kraft lignin, and (c) soda lignin. (d) Corresponding prolate ellipsoid representation from ELLIPS1 for all three lignins and (e) corresponding oblate ellipsoid representation for all three lignins.

Table 2: Viscosity increment for Alcell, kraft, and soda lignins in $90 \%$ DMSO for different values of the hydration or $v_{\mathrm{s}}$ value ( $\mathrm{ml} \mathrm{g}^{-1}$ ).

\begin{tabular}{lrrrrr}
\hline Lignin & $\mathbf{v}$ for $\mathbf{v}_{\mathbf{s}}=\mathbf{0 . 6} \mathbf{~ m ~ g}^{-1}$ & $\mathbf{v}$ for $\mathbf{v}_{\mathbf{s}}=\mathbf{1 . 0} \mathbf{~ m l ~ g}^{-1}$ & $\mathbf{v}$ for $\mathbf{v}_{\mathbf{s}}=\mathbf{1 . 4} \mathbf{~ m l ~ g ~}^{-1}$ & $\mathbf{a} / \mathbf{b}$ (Prolate model) & $\mathbf{a} / \mathbf{b}($ Oblate $\mathbf{m o d e l})$ \\
\hline Alcell & 39.3 & 23.6 & 16.9 & $14 \pm 4$ & $33 \pm 10$ \\
Kraft & 33.8 & 22.7 & 16.2 & $14 \pm 4$ & $32 \pm 8$ \\
Soda & 36.7 & 22.0 & 15.7 & $14 \pm 4$ & $31 \pm 9$ \\
\hline
\end{tabular}

\section{Conclusions}

Implementation of sedimentation velocity and sedimentation equilibrium in the analytical ultracentrifuge provides an alternative to SEC-based procedures for the analysis of the heterogeneity and MW of solubilised lignins. The limitation in the present research is, however, that only $90 \%$ of the lignins were soluble in DMSO. On the other hand, no calibration standards are required and there are no problems related to noninertness of columns as in case of SEC. The MULTISIG and M_INVEQ analyses show the presence of two clear components (major and minor) for Alcell $\mathrm{L}$ and (to a lesser extent) Soda L. For all three lignins investigated in this study, the properties lie within a plausible range, but the MW data obtained by analytical ultracentrifugation are significantly higher than the SEC-based values. A plate shape structure for all three lignins with aspect ratio 30:1 seems to be probable. While the SEC approach is useful in routine work for comparative purposes, modern ultracentrifuge approaches for molecular weight and heterogeneity characterisation for lignins should be considered more in theoretical and practical lignin research.

\section{References}

Ang, S., Rowe, A.J. (2010) Evaluation of the information content of sedimentation equilibrium data in self-interacting systems. Macromol. Biosci. 10:798-807.

Ball, A., Harding, S.E., Mitchell, J.R. (1988) Combined low-speed sedimentation equilibrium/gel permeation chromatography approach to molecular weight distribution analysis. Int. J. Biol. Macromol. 10:259-264. 
Ball, A., Harding, S.E., Simpkin, N.J. (1990) On the molecular weight distribution of dextran T-500. Gums Stabil. Food Ind. 5:447-450.

Baumberger, S., Abaecherli, A., Fasching, M., Gellerstedt, G., Gosselink, R.J.A., Hortling, B., Li, J., Saake, B., de Jong, E. (2007) Molar mass determination of lignins by size-exclusion chromatography: towards standardisation of the method. Holzforschung 61:459-468.

Brebu, M., Vasile, C. (2010) Thermal degradation of lignin: a review. Cellulose Chem. Technol. 44:353-363.

Creeth, J.M., Harding, S.E. (1982) Some observations on a new type of point average molecular weight. J. Biochem. Biophys. Methods 7:25-34.

Creeth, J.M., Pain, R.H. (1967) Prog. Biophys. Mol. Biol. 17:217-287.

Dam, J., Schuck, P. (2004) Determination of sedimentation coefficient distributions by direct modeling of the sedimentation boundary with Lamm equation solutions. Methods Enzymol. 384:185-221.

De Martino, M. (2005) Lignin natural products. Baran Group Meeting. Available from http://www.scripps.edu/baran/ images/grpmtgpdf/Demartino_Nov_05.pdf. Accessed July 3, 2012.

Dong, D., Fricke, A.L. (1995) Intrinsic viscosity and the molecular weight of kraft lignin. Polymer 36:2075-2078.

Favis, B.D., Goring, D.A. I. (1984) A model for the leaching of lignin macromolecules from pulp fibres. J. Pulp Pap. Sci. 10: J139-J143.

Fengel, D., Wegener, G. Wood - chemistry, ultrastructure, reactions. Walter de Gruyter, Berlin and New York, 1984.

García de la Torre, J., Harding, S.E. (2013) Hydrodynamic modelling of protein conformation in solution: ELLIPS and HYDRO. Biophys. Rev. 5:195-206.

Garver, T.M., Callaghan, P.T. (1991) Hydrodynamics of kraft lignins. Macromolecules 24:220-230.

Gillis, R.B., Adams, G.G., Heinze, T., Nikolajski, M., Harding, S.E., Rowe, A.J. (2013) MultiSig: a new high precision approach to the analysis of complex biomolecular systems. Eur. Biophys. J. 42:777-786.

Goring, D.A.I. (1962) The physical chemistry of lignin. Pure Appl. Chem. 5:233-310.

Gosselink, R.J.A., van Dam, J.E.G., de Jong, E., Scott, E.L., Sanders, J.P.M., Li, J., Gellerstedt, G. (2010) Fractionation, analysis, and PCA modeling of properties of four technical lignins for prediction of their application potential in binders. Holzforschung 64:193-200.

Gupta, P.R., Goring, D.A.I. (1960). Physicochemical studies of alkali lignins: iii. size and shape of the macromolecule. Can. J. Chem. 38:270-279.

Harding, S.E. (1997) The intrinsic viscosity of biological macromolecules. Progress in measurement, interpretation and application to structure in dilute solution. Prog. Biophys. Mol. Biol. 68:207-262.

Harding, S.E. (2005) Analysis of polysaccharide size, shape and interaction. In: Analytical Ultracentrifugation: Techniques and Methods. Eds. Scott, D.J., Harding, S.E., Rowe, A.J. Royal Society of Chemistry, Cambridge, UK. pp. 231-252.

Harding, S.E, Dampier, M.J., Rowe, A.J. (1982). The viscosity increment for ellipsoids of revolution: some observations on the Simha formula. Biophys. Chem. 15:205-208.
Harding, S.E., Horton, J.C., Cölfen, H. (1997) The ELLIPS suite of macromolecular conformation algorithms. Eur. Biophys. J. 25:347-359.

Harding, S.E., Cölfen, H., Aziz, Z. (2005) The ELLIPS suite of whole-body protein conformation algorithms for Microsoft Windows. In: Analytical Ultracentrifugation. Techniques and Methods. Eds. Scott, D.J., Harding, S.E., Rowe, A.J. Royal Society of Chemistry, Cambridge, UK. pp. 460-483.

Heinze, T., Nikolajski, M., Daus, S., Besong, T.M.D., Michaelis, N., Berlin, P., Morris, G.A., Rowe, A.J., Harding, S.E. (2011) Proteinlike oligomerisation of carbohydrates. Ang. Chem. Int. Ed. 50:8602-8604.

Käuper, P. (2004) From production to product: Part 1. Solution states of alkaline bagasse lignin solution. Ind. Crops Prod. 20:151-157.

Kubo, S., Kadla, J.F. (2004) Poly(ethylene oxide)/organosolv lignin blends: relationship between thermal properties, chemical structure, and blend behaviour. Macromolecules 37:6904-6911.

Lallave, M., Bedia, J., Ruiz-Rosas, R., Rodríguez-Mirasol, J., Cordero, T., Otero, J.C., Marquez, M., Barrero, A., Loscertales, I.G. (2007) Filled and hollow carbon nanofibers by coaxial electrospinning of Alcell lignin without binder polymers. Adv. Mat. 19:4292-4296.

Laue, T.M., Shah, B.D., Ridgeway, T.M., Pelletier, S.L. (1992) Computer-aided interpretation of analytical sedimentation data for proteins. In: Analytical Ultracentrifugation in Biochemistry and Polymer Science. Eds. Harding, S.E., Rowe, A.J., Horton, J.C. Royal Society of Chemistry, Cambridge, UK. pp. 90-125.

Nikolajski, M., Adams, G.G., Gillis, R.B., Besong, D.T., Rowe, A.J., Heinze, T. Harding, S.E. (2014) Protein-like fully reversible tetramerisation and super-association of an aminocellulose. Nat. Sci. Rep. 4:3861.

Rowe, A.J. (2011) Ultra-weak reversible protein-protein interactions. Methods 54:157-166.

Rubio, M.A., Pethica, B.A., Zuman, P. (1979) The Interactions of carcinogens and co-carcinogens with lignin and other components of dietary fibre. In: Dietary Fibres: Chemistry and Nutrition. Eds. Inglettm G.E., Falkehagm S.I. Academic Press, London. pp. 251-271.

Sarkanen, K.V., Ludwig, C.H. Eds. Lignins: occurrence, formation, structure and reactions. John Wiley \& Sons, Inc., New York, 1971.

Sarkanen, S., Teller, D.C., Hall, J., McCarthy, J.L. (1981) Lignin. 18. Associative effects among organosolv lignin components. Macromolecules 14:426-434.

Sarkanen, S., Teller, D., Abramowski, E. McCarthy, J. (1982) Kraft lignin component conformation and associated complex configuration in aqueous alkaline solution. Macromolecules 15:1098-1104.

Sarkanen, S., Teller, D.C., Stevens, C.R., McCarthy, J.L. (1984) Lignin. 20. Associative interactions between kraft lignin components. Macromolecules 17:2588-2597.

Schachman, H.K. (1992) Is there a future for the ultracentrifuge? In: Analytical Ultracentrifugation in Biochemistry and Polymer Science. Eds. Harding, S.E., Rowe, A.J., Horton, J.C. Royal Society of Chemistry, Cambridge, UK. pp. 1-15.

Schuck, P., Gillis, R.B., Besong, D., Almutairi, F., Adams, G.G., Rowe, A.J., Harding, S.E. (2014) SEDFIT-MSTAR: molecular weight and molecular weight distribution analysis of polymers 
by sedimentation equilibrium in the ultracentrifuge. Analyst 139:79-92.

Simha, R. (1940) The influence of Brownian motion on the viscosity of solutions. J. Phys. Chem. 44:25-34.

Solomon, O.F., Ciuta, I.Z. (1963) Determination de la viscosite intrinseque de solutions de polymers par une simple determination de la viscosite, J. Appl. Polym. Sci. 6:683-686.
Svedberg, T., Pedersen, K.O. The Ultracentrifuge, Clarendon Press, Oxford, 1940.

Vanholme, R., Demedts, B., Morreel, K., Ralph, J., Boerjan, W. (2010) Lignin biosynthesis and structure. Plant Physiol. 153:895-905.

Zhong, R., Ye, Z.H. (2007) Regulation of cell wall biosynthesis. Curr. Opin. Plant Biol. 10:564-572. 